\title{
Mise au point
}

Dr M. Trutmann, Rédacteur en chef

Certains ont cru comprendre en lisant l'éditorial de M. Yves Guisan paru au no 21 du BMS [1] que celui-ci pensait que la situation des médecins était extrêmement confortable. Il semble que le paragraphe cité ci-dessous ait donné lieu à quelques confusions d'interprétations faute de clarté rédactionnelle suffisante:

«[...] les mises en garde bien intentionnées adressées aux partenaires et aux politiques concernant les difficultés techniques d'application des mesures envisagées pour maîtriser les coûts de la santé n'ont été interprétées que comme des opérations de diversion. Comment d'ailleurs attribuer la moindre crédibilité à ces propos? Qu'on le veuille ou non et quoi qu'on dise, la situation de l'ensemble du corps médical est extrêmement confortable, et somme toute assez peu éloignée de celle de quelques numismates notoires dont les revenus hors normes et les scandales périodiques font la une de la Press People.»

Il est bien entendu que M. Guisan rend par la phrase soulignée en italique l'opinion des partenaires et des politiques et non la sienne.

Il aurait fallu pouvoir lire:

«Qu'on le veuille ou non et quoi qu'on dise, la situation de l'ensemble du corps médical ne pourrait être selon eux qu'extrêmement confortable, et somme toute assez peu éloignée de celle de quelques numismates notoires dont les revenus hors normes et les scandales périodiques font la une de la Press People.»
Tous ceux qui suivent les activités de M. Guisan au comité central de la FMH et au Conseil national savent très bien qu'il se bat contre vents et marées pour faire comprendre que l'écrasante majorité des médecins a un revenu correspondant à celui d'un professeur de gymnase, et de cadre moyen ou supérieur pour la plupart des spécialistes, mais certainement pas la situation dorée qui défraie la chronique.

Somme toute, il faut être de mauvaise foi pour mal comprendre le passage en question. La phrase, prise pour elle seule, peut être interprétée de manières différentes, je le concède, mais dans le contexte de l'ensemble de l'éditorial, elle ne peut être plus claire. Ceux qui ne comprennent pas, ou bien ne savent pas lire, ou bien se trompent de cible en adressant à l'auteur des récriminations destinées à d'autres. Dans la traduction allemande, j'ai utilisé le subjonctif «wie könne man ...», «ob man es wolle oder nicht ...», «die Situation der gesamten Ärzteschaft sei ...»- pour bien faire comprendre que l'auteur rend l'opinion des partenaires et des politiques, et non pas la sienne. Si la phrase en question faisait comprendre le contraire, je ne l'aurais pas traduite de la sorte. De plus, ceux qui à part moi ont reçu l'éditorial en copie par courrier n'auraient certainement pas manqué de réagir, si le texte avait laissé entendre le sens que certains veulent lui prêter maintenant. Or, cela n'a pas été le cas ni dans l'immédiat, ni après publication. Je prends ma part de responsabilité dans la confusion que la publication de cet éditorial a pu créer et je demande à l'auteur de m'en excuser. En effet, j'aurais dû être plus attentif à la lecture du texte et le scruter sur d'éventuels manques de clarté, ceci connaissant l'esprit ô combien pointilleux de certains de nos lecteurs.
1 Guisan Y. Les médecins ne sont-ils que des empêcheurs?

Bull Méd Suisses 2003;84(21):1081. 